\title{
MAXIMUM MODULUS OF SOME EXPRESSIONS OF LIMITED ANALYTIC FUNCTIONS *
}

BY

\section{SŌICHI KAKEYA}

\section{Introduction}

Some years ago I published a paper† on "Upper and lower limits of some quantities regarding analytic functions" and discussed some general theorems by which we could systematize certain problems treated independently by several authors and could solve them completely by means of a single principle. $\neq$

In example 5 of that paper, I found the upper limit of

$$
\left|f(0)+A f^{\prime}(0)\right|
$$

with respect to the functions $f(x)$ which are analytic and numericallyr not greater than 1 in the domain $|x| \leqq 1 . \S$ It was an example of the general problem to find the upper limit of

$$
\left|c_{0} f(0)+c_{1} f^{\prime}(0)+\cdots+c_{n} f^{(n)}(0)\right|
$$

in the same domain of functions $f(x)$. A special case of the problem, namely to find the upper limit of

$$
\left|f(0)+\frac{1}{1 !} f^{\prime}(0)+\cdots+\frac{1}{n !} f^{(n)}(0)\right|,
$$

has been solved very elegantly by Prof. E. Landau.|| But his method is not applicable to the general case (1).

We may naturally generalize problem (1) to that of finding the upper limit of

$$
\begin{aligned}
V(f . c, \alpha) \equiv & \mid c_{10} f\left(\alpha_{1}\right)+c_{11} f^{\prime}\left(\alpha_{1}\right)+\cdots+c_{1 n_{1}} f^{\left(n_{1}\right)}\left(\alpha_{1}\right) \\
& +c_{20} f\left(\alpha_{2}\right)+c_{21} f^{\prime}\left(\alpha_{2}\right)+\cdots+c_{2 n_{2}} f^{\left(n_{2}\right)}\left(\alpha_{2}\right) \\
& +\cdots+c_{m 0} f\left(\alpha_{m}\right)+c_{m 1} f^{\prime}\left(\alpha_{m}\right) \\
& +\cdots+c_{m n_{m}} f^{\left(n_{m}\right)}\left(\alpha_{m}\right) \mid
\end{aligned}
$$

where $\alpha_{1}, \alpha_{2}, \cdots, \alpha_{m}$ are given points within the unit circle $|x|=1$.

* Presented to the Society, February, 1923.

†Science Reports of the Töhoku Imperial University, vol. 6 (1917), p. 153.

$\ddagger$ See the examples given in the end of that paper.

$\$$ Throughout the paper the domain is understood to include the boundary.

\|Archivder Mathematik und Physik, ser. (3), vol. 21 (1913), p. 250, or his work "Darstellung und Begründung einiger neuerer Ergebnisse der Funktionentheorie," p. 20. 
In my paper, above mentioned, I proved that a function $f(x)$ giving the maximum of (2) exists and is a rational function of degree at most $\sum_{i=1}^{m} n_{i}+m-1$, whose modulus is constantly equal to 1 along the unit circle $|x|=10^{*}$ But I have never found any such simple method of getting the required maximum and extremal for this general problem as Professor Landau has found in his special case. The only method I could suggest is to calculate algebraically the maximum of (2) among rational functions of the form

$$
f(x)=e^{i \theta} \frac{\left(x-a_{1}\right)\left(x-a_{2}\right) \cdots\left(x-a_{\kappa}\right)}{\left(\bar{a}_{1} x-1\right)\left(\bar{a}_{2} x-1\right) \cdots\left(\bar{a}_{\kappa} x-1\right)},
$$

$\kappa=\sum n_{i}+m-1, \bar{a}_{i}$ conjugate to $a_{i}, \theta$ real, $\left|a_{1}\right|,\left|a_{2}\right|, \cdots,\left|a_{\kappa}\right| \leqq 1$, which is the general expression of the rational function of degree at most $\kappa$, whose modulus along the unit circle is constantly equal to 1 . We had, however, no further information about the extremal and extreme value thus obtained.

The main aim of this paper is to investigate more fully this extremal and this extremum.

\section{Uniqueness of solution}

It is convenient to prove at first the uniqueness of the extremal.

Our problem is to find the maximum of (2) under the condition that $f(x)$ is analytic and numerically not greater than 1 for $|x| \leqq 1$. Here all the c's are given constants, not zero at the same time, and the $\alpha$ 's are given points within the unit circle, i.e., $\left|\alpha_{i}\right|<1$.

As stated above, there exists at ieast one extremal. Now let us suppose that we have two functions $f_{1}(x)$ and $f_{2}(x)$, both of which satisfy the given conditions and give the same maximum value of $V(f, c, \alpha)$, say $M$.

If we take suitable real constants $\theta_{1}$ and $\theta_{2}$, we can make the functions $e^{i \theta_{1}} f_{1}(x)$ and $e^{i \theta_{2}} f_{2}(x)$, which are also extremals, such that the expression within the sign | | in $V(f, c, \alpha)$ becomes positive when we substitute either of them for $f(x)$. And under these conditions it is evident that

is also an extremal.

$$
\frac{1}{2}\left\{e^{i \theta_{1}} f_{1}(x)+e^{i \theta_{2}} f_{2}(x)\right\}=F(x)
$$

Since $e^{i \theta_{1}} f_{1}(x)$ and $e^{i \theta_{2}} f_{2}(x)$ are numerically not greater than 1 along the unit circle, $F(x)$ must have the same property, and at any point on the circle, where the two functions $e^{i \theta_{1}} f_{1}(x)$ and $e^{i \theta_{2}} f_{2}(x)$ are unequal, it must have a modulus less than 1.

On the other hand, I have proved $\dagger$ that, among the functions $f(x)$ which

* This is a special case of Cor. $3 b$, p. 161 , of my paper cited before.

†Science Reports of the Tohoku Imperial University, vol. 4 (1915), p. 297. This was published in August, 1915. A few months later I saw a paper by Professor Pick, in $M$ a t h e $\mathrm{m}$ a $\mathrm{t}$ i $\mathrm{s}$ c h e $\mathrm{A} \mathrm{n}$ a a l e $\mathrm{n}$, vol. 77, regarding a similar character of analytic functions. It was published in December, 1915. 
are analytic for $|x| \leqq 1$ and satisfy the conditions

$$
\begin{aligned}
& f\left(\alpha_{1}\right)=F\left(\alpha_{1}\right), \quad f^{\prime}\left(\alpha_{1}\right)=F^{\prime}\left(\alpha_{1}\right), \cdots, f^{\left(n_{1}\right)}\left(\alpha_{1}\right)=F^{\left(n_{1}\right)}\left(\alpha_{1}\right) \\
& f\left(\alpha_{m}\right)=F\left(\alpha_{m}\right), \quad f^{\prime}\left(\alpha_{m}\right)=F^{\prime}\left(\alpha_{m}\right), \quad \cdots, f^{\left(n_{m}\right)}\left(\alpha_{m}\right)=F^{\left(n_{m}\right)}\left(\alpha_{m}\right),
\end{aligned}
$$

there is only one function $f_{0}(x)$ whose maximum modulus for $|x| \leqq 1$ is less than that of any other function. $f_{0}(x)$ is a rational function of degree at most $\kappa=\sum n_{i}+m-1$ and is of constant modulus, $N$ say, along the unit circle $|x|=1$.

Now the quantity $N$ can not be greater than or equal to 1 , for the function $F(x)$ itself, whose modulus is not greater than 1 along the unit circle and is at least less than 1 at some point on the circle, and therefore is different from $f_{0}(x)$, satisfies the same condition (4). Let us now suppose that $N$ is less than 1 . Since $f_{0}(x)$ gives the value $M$ of $V(f, c, \alpha)$ when we substitute it for $f(x)$, we can evidently find a sufficiently small variation of $f_{0}(x)$, say $\delta f_{0}(x)$, such that

$$
\left|f_{0}(x)+\delta f_{0}(x)\right|<1 \quad \text { for } \quad|x| \leqq 1
$$

and the expression (2) becomes greater than $M$ when we substitute $f_{0}(x)+\delta f_{0}(x)$ for $f(x)$, for (2) is a linear function of $f(x)$ and its derivatives. Hence $M$ is not the maximum and we have a contradiction.

The only remaining possibility is that $e^{i \theta_{1}} f_{1}(x)$ and $e^{i \theta_{2}} f_{2}(x)$ be identically equal along the unit circle, and hence within the circle. Hence we see that $f_{1}(x)$ and $f_{2}(x)$ can differ at most by a constant factor whose modulus is 1 .

Conversely if $f_{1}(x)$ is an extremal of our problem, any function $f_{2}(x)$ which differs from $f_{1}(x)$.only by a constant factor of modulus 1 is evidently an extremal.

By combining the results of the earlier paper with those of the present section we have the following

THEOREM: Let $\{f(x)\}$ be the set of all functions $f(x)$ which in the closed domain $|x| \leqq 1$ are analytic and of absolute value not exceeding unity. Let $c_{10}, c_{11}, \cdots c_{1 n_{1}} ; c_{20}, c_{21}, \cdots c_{2 n_{2}} ; \cdots ; c_{m 0}, c_{m 1}, \cdots c_{m n_{m}}$ be a set of given (complex) constants not all of which are zero. Let $\alpha_{1}, \alpha_{2}, \cdots \alpha_{m}$ be a set of given complex constants each of absolute value less than unity. Let

$$
\begin{aligned}
V(f, c, \alpha) & \equiv \mid c_{10} f\left(\alpha_{1}\right)+c_{11} f^{\prime}\left(\alpha_{1}\right)+\cdots+c_{1 n_{1}} f^{\left(m_{1}\right)}\left(\alpha_{1}\right)+c_{20} f\left(\alpha_{2}\right) \\
& +c_{21} f^{\prime}\left(\alpha_{2}\right)+\cdots+c_{2 m_{2}} f^{\left(m_{3}\right)}\left(\alpha_{2}\right)+\cdots+c_{m 0} f\left(\alpha_{m}\right) \\
& \quad+c_{m 1} f^{\prime}\left(\alpha_{m}\right)+\cdots+c_{m n_{m}} f^{\left(m_{m}\right)}\left(\alpha_{m}\right) \mid .
\end{aligned}
$$

Then: (1) There exists at least one function $f_{0}(x)$ iu the set $\{f(x)\}$ such that $V\left(f_{0}, c, \alpha\right)=M$, the least upper bound of $V(f, c, \alpha)$ on $\{f(x)\}$. 
(2) $f_{0}(x)$ is a rational function of degree

at most, is of form

$$
\kappa=m-1+\sum_{i=1}^{i=m} n_{i}
$$

$$
f_{0}(x) \equiv e^{i \theta} \frac{\left(x-a_{1}\right)\left(x-a_{2}\right) \cdots\left(x-a_{k}\right)}{\left(\bar{a}_{1} x-1\right)\left(\bar{a}_{2} x-1\right) \cdots\left(\bar{a}_{k} x-1\right)}
$$

( $\bar{a}_{i}$ conjugate to $a_{i},\left|a_{i}\right| \leqq 1, i=1,2, \cdots \kappa, \theta$ real and constant); and on the periphery $|x|=1$ of the unit circle is of constant absolute value unity.

(3) $f_{0}(x)$ is uniquely determined except for the constant factor $e^{i \theta}$ of unit absolute value.

(4) If $\theta$ is any real number, and

then

$$
F_{0}(x) \equiv e^{i \theta} f_{0}(x)
$$

$$
V\left(F_{0}, c, \alpha\right)=V\left(f_{0}, c, \alpha\right)=M \text {. }
$$

\section{Transformation of the problem}

We will now put the problem into a more convenient form.

Since the function $f(x)$ under consideration is analytic in the unit circle and all the points $\alpha_{1}, \alpha_{2}, \cdots, \alpha_{m}$ lie within the unit circle, we have

$$
f^{(p)}\left(\alpha_{i}\right)=\frac{p !}{2 \pi i} \int_{C} \frac{f(x)}{\left(x-\alpha_{i}\right)^{p+1}} d x,
$$

$C$ denoting that the integration is effected along the unit circle. Hence the expression $V(f, c, \alpha)$ can be written in the form

where

$$
V(f, c, \alpha)=I \equiv \int_{C} \frac{Q(x)}{P(x)} f(x) d x,
$$

$$
P(x)=\left(x-\alpha_{1}\right)^{n_{1}+1}\left(x-\alpha_{2}\right)^{n_{2}+1} \cdots\left(x-\alpha_{m}\right)^{n_{m}+1}
$$

and $Q(x)$ is a polynomial at most of degree $\kappa=\sum n_{i}+m-1$. Our problem is, then, equivalent to that of finding the maximum modulus of the integral $I$.

The integral $I$ is evidently equal to

$$
J \equiv \int_{C}\left\{\frac{Q(x)}{P(x)}+\varphi(x)\right\} f(x) d x,
$$

where $\varphi(x)$ is an arbitrary function which is analytic in the unit circle. So our problem is to find the maximum modulus of $J$ which is known to be invariant with respect to the form of $\varphi(x)$.

Any function which is regular in the unit circle except for $\kappa+1$ poles can be put in the form $Q(x) / P(x)+\varphi(x)$. Hence our problem is equivalent 
to that of finding the maximum modulus of

$$
\int_{C} R(x) f(x) d x
$$

$R(x)$ being a given function which is meromorphic within the unit circle and regular along the circle.

For the sake of simplicity, we will put hereafter

and

$$
\sum n_{i}+m=\kappa+1=n
$$

$$
P(x)=\left(x-\alpha_{1}\right)\left(x-\alpha_{2}\right) \cdots\left(x-\alpha_{n}\right)
$$

where $\left|\alpha_{i}\right|<1$, and let $Q(x)$ denote a given polynomial of degree at most $n-1$. If we make some of the $\alpha$ 's equal to one another, then we can get the form (6).

\section{Solution of the problem}

In our problem, we can take as $\varphi(x)$ any function analytic in the unit circle.

Now assume that we could find a function $\varphi(x)$ and a set of constants $a_{1}, a_{2}, \cdots, a_{m}$, which are such that the function

where

$$
x\left\{\frac{Q(x)}{P(x)}+\varphi(x)\right\} \prod_{i=1}^{m} \frac{x-a_{i}}{\bar{a}_{i} x-1},
$$

$$
m \leqq n-1, \quad\left|a_{i}\right|<1 \quad \text { for } \quad i=1,2, \cdots, m,
$$

has a constant amplitude along the unit circle $|x|=1$. Then we can easily see that the required maximum of $I$ or $J$ is

(11) $\quad M=\left|\int_{C}\left\{\frac{Q(x)}{P(x)}+\varphi(x)\right\} \prod_{i=1}^{m} \frac{x-a_{i}}{\bar{a}_{i} x-1} d x\right|=\mid \int_{C} \frac{Q(x)}{P(x)} \prod_{i=1}^{m} \frac{x-a_{i}}{\bar{a}_{i} x-1} d x$

and the required extremal is

$$
f_{0}(x)=e^{i \theta} \prod_{i=1}^{m} \frac{x-a_{i}}{\bar{a}_{i} x-1}
$$

For, in this case, the function $f_{0}(x)$ is analytic in the unit circle by virtue of (10) and is of constant modulus 1 along the circle, so $M$ is one of the absolute values of the form (5) or (7); and moreover, taking any function $f(x)$ analytic and numerically not greater than 1 for $|x| \leqq 1$, we have, putting $x=e^{i \sigma}$ for the integration,

$$
\begin{aligned}
\left|\int_{C}\left\{\frac{Q(x)}{P(x)}+\varphi(x)\right\} f(x) d x\right| & \leqq \int_{0}^{2 \pi}\left|x\left\{\frac{Q(x)}{P(x)}+\varphi(x)\right\}\right| d \sigma \\
& =\int_{0}^{2 \pi}\left|x\left\{\frac{Q(x)}{P(x)}+\varphi(x)\right\} \prod_{i=1}^{m} \frac{x-a_{i}}{\bar{a}_{i} x-1}\right| d \sigma ;
\end{aligned}
$$


but since the expression of the last integrand has a constant amplitude throughout the integration we have the last integral

$$
\begin{aligned}
& =\left|\int_{0}^{2 \pi} x\left\{\frac{Q(x)}{P(x)}+\varphi(x)\right\} \prod_{i=1}^{m} \frac{x-a_{i}}{\bar{a}_{i} x-1} d \sigma\right| \\
& =\left|\int_{C}\left\{\frac{Q(x)}{P(x)}+\varphi(x)\right\} \prod_{i=1}^{m} \frac{x-a_{i}}{\bar{a}_{i} x-1} d x\right|=M .
\end{aligned}
$$

Now since $\left|\alpha_{i}\right|<1$, the function $\left[\prod_{i=1}^{n}\left(\bar{\alpha}_{i} x-1\right)\right]^{-1}$ must be analytic in the unit circle. Hence if the remainder and the quotient of $Q(x) \prod\left(\bar{\alpha}_{i} x-1\right)$ divided by $P(x)$ are $R(x)$ and $S(x)$ respectively, then for any function $\psi(x)$ which is analytic in the unit circle we can find uniquely the function $\varphi(x)$ which is also analytic in the unit circle and satisfies the equation

$$
Q(x)+P(x) \varphi(x)=\frac{R(x)+P(x) \psi(x)}{\prod\left(\bar{\alpha}_{i} x-1\right)} .
$$

The function $\varphi(x)$ is to be found from

$$
\varphi(x)=\frac{\psi(x)-S(x)}{\prod\left(\bar{\alpha}_{i} x-1\right)} .
$$

On the other hand, we have the theorem, which I will prove in the next chapter, that there exists a function $\psi(x)$ analytic in the unit circle, which satisfies a relation of the form

$$
R(x)+P(x) \psi(x)=c \prod_{i=1}^{m}\left(\bar{a}_{i} x-1\right)^{2} \prod_{i=1}^{n-m-1}\left(x-b_{i}\right)\left(\bar{b}_{i} x-1\right)
$$

where $c$ and the $b_{i}$ 's are constants, the $a_{i}^{\prime}$ 's are constants numerically less than 1 , and $m$ does not exceed $n-1$.

If we construct the function $\varphi(x)$ from the above function $\psi(x)$ by the formula (14), then we get, after a short calculation,

$$
\begin{aligned}
x\left\{\frac{Q(x)}{P(x)}+\varphi(x)\right\} \prod^{m} \frac{x-a_{i}}{\bar{a}_{i} x-1} \\
=c \frac{x \prod^{m}\left(x-a_{i}\right)\left(\bar{a}_{i} x-1\right){ }^{n-m-1}\left(x-b_{i}\right)\left(\bar{b}_{i} x-1\right)}{\prod^{n}\left(x-\alpha_{i}\right)\left(\bar{\alpha}_{i} x-1\right)} .
\end{aligned}
$$

But the variation of amplitude of the quadratic function which has the form $(x-t)(\bar{t} x-1)$ along the unit circle $|x|=1$ is equal to that of $x$. Hence the variation of amplitude of the right-hand member of (16) is zero, i.e., the function has constant amplitude along the unit circle.

Hence we see that there exists always a function $\varphi(x)$ and a set of constants $a_{i}$ under the required conditions, which causes the left-hand member 
of (16), namely the expression (9), to have constant amplitude along the unit circle. The $\varphi(x)$ and the $a_{i}$ are determined by (14) and (15). Thus the problem is solved theoretically.

But I can only prove, in the next chapter, the existence of $\psi(x), a_{i}, b_{i}$ and $c$ of (15), without knowing any simple and practical method of finding them. So, in this respect, our problem is not yet completely solved.

\section{Proof of auxiliary theorem}

We are now to prove the theorem about the relation (15). At first we will assume that all the $\alpha$ 's are different.

The left-hand side of (15) is $R(x)+P(x) \psi(x)$, where $\psi(x)$ is an arbitrary function analytic for $|x| \leqq 1, R(x)$ is a given polynomial of degree $n-1$ at most, and $P(x)$ is a given polynomial of degree $n$, whose zeros are all within the unit circle. This is merely the general expression of the function $F(x)$ analytic for $|x| \leqq 1$, which satisfies the conditions

$$
F\left(\alpha_{i}\right)=R\left(\alpha_{i}\right), \quad i=1,2, \cdots, n .
$$

Therefore we can state as follows the theorem to be proved:

Let $\alpha_{1}, \alpha_{2}, \cdots, \alpha_{n}$ be $n$ different points within the unit circle $|x|=1$ and $u_{1}, u_{2}, \cdots, u_{n}$ be $n$ given constants. Then there exists a function of the form

$$
\begin{gathered}
F(x)=c \prod_{i=1}^{m}\left(\bar{a}_{i} x-1\right)^{2} \prod_{i=1}^{n-m-1}\left(x-b_{i}\right)\left(\bar{b}_{i} x-1\right), \\
m \leqq n-1, \quad\left|a_{i}\right|<1, \quad \text { for } \quad i=1,2, \cdots, m,
\end{gathered}
$$

which satisfies the conditions

$$
F\left(\alpha_{i}\right)=u_{i}, \quad i=1,2,3, \cdots n .
$$

In other words two sets of constants $\left\{a_{i}\right\},\left\{b_{i}\right\}$, one constant $c$, and one integer $m$ can be found satisfying the above relations. The function $F(x)$ thus obtained is unique.

If there exists one function (17), then the function

$$
\prod_{i=1}^{m} \frac{x-a_{i}}{\bar{a}_{i} x-1}
$$

determined by $a_{i}$ is an extremal of the corresponding problem of the preceding chapter, $Q(x)$ and $R(x)$ being the polynomials of degree at most $n-1$ determined from (13) and (15) or from the relations

$$
R\left(\alpha_{i}\right)=u_{i}, \quad Q\left(\alpha_{i}\right)=\frac{u_{i}}{\prod_{k=1}^{n}\left(\bar{\alpha}_{k} \alpha_{i}-1\right)} .
$$


Such an extremal is known to be unique save for a factor of the form $e^{i \theta}$. Hence if there are two functions $F_{1}(x)$ and $F_{2}(x)$ of the form (17) satisfying (18) and (19), they must have common values for $a_{i}$. So we have the relation of the form

and

$$
T(x)=\frac{F_{1}(x)}{F_{2}(x)}=c \prod_{i=1}^{n-m-1} \frac{\left(x-b_{i 1}\right)\left(\bar{b}_{i 1} x-1\right)}{\left(x-b_{i 2}\right)\left(\bar{b}_{i 2} x-1\right)}
$$

$$
T\left(\alpha_{i}\right)=1, \quad i=1,2, \cdots, n .
$$

If some of the $u_{i}$ 's, say $u_{n}, u_{n-1}, \cdots, u_{\nu}$, are equal to zero, then the corresponding $n-\nu+1$ of the $b_{i 1}$ 's are equal to $n-\nu+1$ of the $b_{i 2}$ 's respectively, and hence we are to take $\nu-1$ instead of $n$ in the above relation. Here as before we see that $T(x)$ has constant amplitude, say $\omega$, along the unit circle.

Let us suppose that $\left|b_{i 2}\right| \neq 1, i=1,2, \cdots, n-m-1$, and that $\omega \neq 0$. Since $r e^{i \omega}-1$ can not change its amplitude by any angle more than or equal to $\pi$ by means of the variation of the positive quantity $r$, the function

$$
T(x)-1=\prod^{n-m-1} \frac{\left(x-b_{i 1}\right)\left(\bar{b}_{i 1} x-1\right)}{\left(x-b_{i 2}\right)\left(\bar{b}_{i 2} x-1\right)}-1
$$

can not change its amplitude when $x$ makes a complete revolution along the unit circle. But, on the other hand, the function (20) can have only $n-m-1$ poles $b_{i 2}$ and has at least $n$ zeros $\alpha_{i}$ within the unit circle, so that the change of amplitude must be at least $2(m+1) \pi$. Thus we are led to an absurdity.

In the case when $\omega=0$, we can also establish a similar contradiction, taking the function $T(x)-\epsilon$, where $\epsilon$ is some complex number sufficiently near to 1 such that the function $T(x)-\epsilon$ has at least $n$ zeros sufficiently near to $\alpha_{i}$. In the case where some of the $\left|b_{i 2}\right|$ are equal to 1 , we have again a similar absurdity by making those $\left|b_{i 2}\right|$ so little smaller than 1 that the transformed function has at least $n$ zeros sufficiently near to $\alpha_{i}$.

The only remaining possibility, then, is that $T(x)-1$ is identically zero, namely $F_{1}(x) \equiv F_{2}(x)$. Hence we see that the function of the form (17) must be unique in case it exists.

Here we mean the uniqueness of the function $F(x)$ in (17), but the constants $c, a_{i}, b_{i}$ are not determined separately; for example we can interchange the values of $a_{1}, a_{2}$ or of $b_{1}, b_{2}$.

We have identically

$$
\left(x-b_{i}\right)\left(\bar{b}_{i} x-1\right)=b_{i} \bar{b}_{i}\left(x-\frac{1}{\bar{b}_{i}}\right)\left(\frac{1}{b_{i}} x-1\right),
$$

and therefore taking one or the other form of factors we can always make

$$
\left|b_{i}\right| \leqq 1, \quad i=1,2, \cdots, n-m-1
$$

in the expression (17). We assume this relation hereafter. 
Under these circumstances, the constant $c$ and the two aggregates $\left\{a_{i}\right\}$, $\left\{b_{i}\right\}$ are determined separately in case they exist, except for the case where $c=0$, namely

$$
u_{1}=u_{2}=\cdots=u_{n}=0 .
$$

If we take $\rho u_{i}$ instead of $u_{i}$, the corresponding $\left\{a_{i}\right\},\left\{b_{i}\right\}$ are invariant and $c$ becomes $\rho c$. Hence we see that $\left\{a_{i}\right\},\left\{b_{i}\right\}$ are perfectly determined in case they exist, when we give the ratios

$$
u_{1}: u_{2}: \cdots: u_{n}
$$

or, as usual, the sequence of values

where

$$
\frac{u_{1}}{r}, \frac{u_{2}}{r}, \cdots, \frac{u_{n}}{r}
$$

$$
r=\left\{\left|u_{1}\right|^{2}+\left|u_{2}\right|^{2}+\cdots+\left|u_{n}\right|^{2}\right\}^{2} .
$$

We now consider a Riemann surface $S$ consisting of two overlapping unit circles connected along their whole peripheries, the common periphery being supposed to belong to the second sheet. Mark the numbers $a_{i}$ on the first sheet and the numbers $b_{i}$ on the second sheet. Then we get an aggregate of $n-1$ points on $S$ corresponding to the ratios (23) or to the sequence (24). The ratios (23) vary continuously as the corresponding aggregate $\left\{t_{i}\right\}$ on $S$ varies continuously. For any factor of (17) is continuous with respect to $a_{i}$ or $b_{i}$, and when $a_{i}$ on the first sheet comes to $b_{i}$ on the second sheet passing through the point $t(|t|=1)$ on the periphery the factor $(\bar{t} x-1)^{2}$ suddenly changes to $(x-t)(\bar{t} x-1)=t(\bar{t} x-1)^{2}$ at the moment of passing the periphery, so that all $u_{i}$ 's are only multiplied by a common factor $t$, the ratios remaining unchanged.

Conversely, if we suppose that $\left\{t_{i}\right\}$ on $S$ always exists corresponding to the given ratios (23), then it must vary continuously as the given ratios (23) vary continuously. For in the contrary case, we must have at least two different limiting aggregates $\left\{t_{i}^{\prime}\right\},\left\{t_{i}^{\prime \prime}\right\}$ to which $\left\{t_{i}\right\}$ tends when the sequence (24) tends to a fixed sequence $\left\{u_{i} / r\right\}$ at which $\left\{t_{i}\right\}$ is discontinuous. But since the sequence (24) varies continuously with $\left\{t_{i}\right\}$ we must have then the two aggregates $\left\{t_{i}^{\prime}\right\}$ and $\left\{t_{i}^{\prime \prime}\right\}$ both of which correspond to the same sequence $\left\{u_{i} / r\right\}$. This is incompatible with the unique determination of $\left\{a_{i}\right\},\left\{b_{i}\right\}$ by means of $\left\{u_{i} / r\right\}$. $^{*}$

If we now put

$$
\begin{aligned}
& E(x, t)=(\bar{t} x-1)^{2} \text { when } t \text { is on the first sheet of } S, \text { and } \\
& E(x, t)=(x-t)(\bar{t} x-1) \text { when } t \text { is on the second sheet of } S,
\end{aligned}
$$

* For the general theorem relating to this property, see Pierpont's Theory of Functions of Real Variables, II, p. 609. 
then the theorem to be proved is that there is one and only one function of the form

under the conditions

$$
F(x)=c \prod_{i=1}^{n-1} E\left(x, t_{i}\right)
$$

$$
F\left(\alpha_{i}\right)=u_{i}, \quad i=1,2, \cdots, n .
$$

I will prove this by mathematical induction assuming the theorem for the case $n-1$. It is evident that we can assume in our proof, without loss of generality, that every $u_{i}, i=1,2, \cdots, n$, is not zero.

By assumption, there exists one and hence only one function of the form

$$
G(x)=c \prod_{i=1}^{n-2} E\left(x, t_{i}\right)=c H(x)
$$

when we have the condition

$$
G\left(\alpha_{i}\right)=\frac{u_{i}}{E\left(\alpha_{i}, t\right)}, \quad i=1,2, \cdots, n-1 .
$$

$I_{n}$ other words an aggregate $\left\{t_{1}, t_{2}, \cdots, t_{n-2}\right\}$ is uniquely determined in terms of the parameter $t$ when we require that the ratios

shall be equal to

$$
\frac{u_{1}}{E\left(\alpha_{1}, t\right)}: \frac{u_{2}}{E\left(\alpha_{2}, t\right)}: \cdots: \frac{u_{n-1}}{E\left(\alpha_{n-1}, t\right)}
$$

Now if the function

$$
K(t)=H\left(\alpha_{n}\right) E\left(\alpha_{n}, t\right) \frac{u_{1}}{H\left(\alpha_{1}\right) E\left(\alpha_{1}, t\right)}=u_{1} \frac{E\left(\alpha_{n}, t\right) \prod^{n-2} E\left(\alpha_{n}, t_{i}\right)}{E\left(\alpha_{1}, t\right) \prod^{n-2} E\left(\alpha_{1}, t_{i}\right)}
$$

should become equal to $u_{n}$ for some value of $t$, say $t=t_{n-1}$, then for the corresponding $\left\{t_{i}\right\}$ we have

or

$$
\frac{u_{1}}{H\left(\alpha_{1}\right) \dot{E}\left(\alpha_{1}, t_{n-1}\right)} H\left(\alpha_{n}\right)=\frac{u_{n}}{E\left(\alpha_{n}, t_{n-1}\right)}
$$

Hence if we put

$$
G\left(\alpha_{n}\right)=\frac{u_{n}}{E\left(\alpha_{n}, t_{n-1}\right)}
$$

then

$$
F(x)=G(x) E\left(x, t_{n-1}\right)=c \prod_{i=1}^{n-1} E\left(x, t_{i}\right),
$$

$$
F\left(\alpha_{i}\right)=u_{i}, \quad i=1,2, \cdots, n ;
$$

and the theorem is therefore proved for the case $n$.

Now every term of the ratios (25) is evidently continuous with respect to 
$t$ on the surface $S$ except at the points $\alpha_{1}, \alpha_{2}, \cdots, \alpha_{n-1}$ on the second sheet and at the points on the common periphery of the circular sheets. If $t$ tends to the periphery from the first sheet, the limiting ratio is

$$
\frac{u_{1}}{\left(\bar{t} \alpha_{1}-1\right)^{2}}: \frac{u_{2}}{\left(\bar{t} \alpha_{2}-1\right)^{2}}: \cdots: \frac{u_{n-1}}{\left(\bar{t} \alpha_{n-1}-1\right)^{2}} ;
$$

and if $t$ comes from the second sheet the ratio is

$$
\frac{u_{1}}{\left(\alpha_{1}-t\right)\left(\bar{t} \alpha_{1}-1\right)}: \frac{u_{2}}{\left(\alpha_{2}-t\right)\left(\bar{t} \alpha_{2}-1\right)}: \cdots: \frac{u_{n-1}}{\left(\alpha_{n-1}-t\right)\left(\bar{t} \alpha_{n-1}-1\right)}
$$

which is equal to

$$
\frac{u_{1}}{t\left(\bar{t} \alpha_{1}-1\right)^{2}}: \frac{u_{2}}{t\left(\bar{t} \alpha_{2}-1\right)^{2}}: \cdots: \frac{u_{n-1}}{t\left(\bar{t} \alpha_{n-1}-1\right)^{2}},
$$

since $|t|=1$. These two ratios are equal. Hence the ratio (25) is continuous when $t$ passes the periphery from the first sheet to the second.

When $t$ approaches $\alpha_{i}$ on the second sheet, $i$ being one of the numbers $1,2,3, \cdots, n-1$, the $i$ th term of the ratio (25) tends to infinity while the other terms remain finite, namely the $i$ th term $H\left(\alpha_{i}\right)$ of the ratio (26) remains finite while the other terms $H\left(\alpha_{1}\right), H\left(\alpha_{2}\right), \cdots, H\left(\alpha_{i-1}\right), H\left(\alpha_{i+1}\right), \cdots$, $H\left(\alpha_{n-1}\right)$ tend to zero. Therefore the aggregate $\left\{t_{1}, t_{2}, \cdots, t_{n-2}\right\}$ must tend to the aggregate $\left\{\alpha_{1}, \alpha_{2}, \cdots, \alpha_{i-1}, \alpha_{i+1}, \cdots, \alpha_{n-1}\right\}$ of the second sheet as a whole; and consequently $H\left(\alpha_{i}\right), H\left(\alpha_{n}\right), E\left(\alpha_{n}, t\right)$ tend respectively to some finite constants not equal to zero. When $t$ makes a complete positive revolution along a sufficiently small circle around the point $\alpha_{i}$ on the second sheet, $i$ being one of $2,3, \cdots, n-1$, then the fraction

$$
\frac{u_{1}}{E\left(\alpha_{1}, t\right)} / \frac{u_{i}}{E\left(\alpha_{i}, t\right)}=\frac{H\left(\alpha_{1}\right)}{H\left(\alpha_{i}\right)}
$$

makes a complete positive revolution along a sufficiently small circuit around the origin. And, since $H\left(\alpha_{i}\right)$ remains almost fixed and not zero, $H\left(\alpha_{1}\right)$ makes a complete positive revolution along a sufficiently small circuit around the origin. Hence the function $K(t)$ makes a negative revolution around the origin describing approximately a large circle. The same variation of $K(t)$ can be seen when $t$ makes a positive revolution around the point $\alpha_{1}$ on the second sheet, since $K(t)$ has the factor $\left[E\left(\alpha_{1}, t\right)\right]^{-1}$. Lastly if $t$ tends to $\alpha_{n}$ on the second sheet, no term of (26) can approach zero, and $K(t)$ which has the factor $E\left(\alpha_{n}, t\right)$ must tend to zero.

The function $K(t)$ is continuous unless $t$ approaches some one of the points $\alpha_{1}, \alpha_{2}, \cdots, \alpha_{n-1}$. For under these circumstances $\left\{t_{i}\right\}$ is continuous as I have said before, $H\left(\alpha_{1}\right)$ and $E\left(\alpha_{1}, t\right)$ do not approach zero, and when $t$ or $t_{i}$ 
passes through the periphery of $S$, the denominator and numerator of $K(t)$ are multiplied by the same factor $t$ or $t_{i}$ at the same time.

Now let $t$ move positively along the $n-1$ loops around $\alpha_{1}, \alpha_{2}, \cdots, \alpha_{n-1}$ of the second sheet of $S$, which consist of $n-1$ sufficiently small circles each around one of the $\alpha_{1}, \alpha_{2}, \cdots, \alpha_{n-1}$ of the second sheet and $n-1$ double paths radiating from some one point (Fig. I). Then the corresponding path of $K(t)$ on the ordinary complex plane consists of $n-1$ large approximate
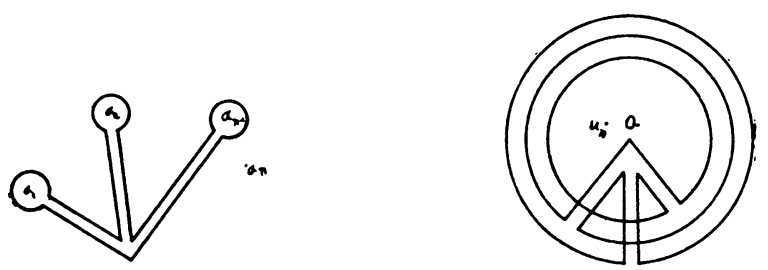

circles around the origin and $n-1$ double paths radiating from some one point (Fig. II). By a small variation of the double path, if necessary, we can make the given point $u_{n}$ lie within the path II.

We can make now the loop path I vary continuously on the Riemann surface $S$ without passing through any of the points $\alpha_{1}, \alpha_{2}, \cdots, \alpha_{n-1}$ on the second sheet so that ultimately it reduces to a point $\alpha_{n}$, in which case the corresponding path II must reduce to the origin. During this continuous variation of the path II, it must pass in some moment the point $u_{n}$. In other words, we must have some value of $t$, say $t=t_{n-1}$, for which $K(t)=u_{n}$. Thus the theorem is proved, in virtue of the preceding remark, for the case $n$.

The theorem is evidently true when $n=1$, and therefore, by the above mathematical induction, our theorem is proved, for the case where all $\alpha$ 's are different.

Next we can easily extend the theorem to the general case where some of the $\alpha$ 's are equal, namely the case where the function $P(x)$ has multiple zeros, say

$$
\begin{gathered}
\left(n_{\kappa}+1\right) \text { uple zeros } \alpha_{\kappa}, \quad \kappa=1,2, \cdots, p, \\
\sum n_{\kappa}+p=n .
\end{gathered}
$$

In this case, the expression $R(x)+P(x) \psi(x)$ is the general form of an analytic function $F(x)$ for $|x| \leqq 1$, whose derivatives at $\alpha_{k}$ are given as follows:

$F\left(\alpha_{1}\right)=R\left(\alpha_{1}\right), \quad F^{\prime}\left(\alpha_{1}\right)=R^{\prime}\left(\alpha_{1}\right), \quad \cdots, \quad F^{\left(n_{1}\right)}\left(\alpha_{1}\right)=R^{\left(n_{1}\right)}\left(\alpha_{1}\right)$,
$\left.\dot{F} \cdot \cdot \cdot \alpha_{p}\right)=R\left(\alpha_{p}\right), \quad \cdot F^{\prime}\left(\alpha_{p}\right)=R^{\prime}\left(\alpha_{p}\right), \quad \cdots, \quad F^{\left(n_{p}\right)}\left(\alpha_{p}\right)=R^{\left(n_{p}\right)}\left(\alpha_{p}\right)$.

Our extended theorem can then be stated as follows: 
There exists one and only one function of the form

$$
\begin{aligned}
& F(x)=c \prod_{i=1}^{m}\left(\bar{a}_{i} x-1\right)^{2} \prod_{i=1}^{n-m-1}\left(x-b_{i}\right)\left(\bar{b}_{i} x-1\right), \\
& 0 \leqq m \leqq n-1, \quad\left|a_{i}\right|<1, \quad i=1,2, \cdots, m,
\end{aligned}
$$

under the condition,

$$
\begin{array}{ccc}
F\left(\alpha_{\kappa}\right)=u_{\kappa}, \quad F^{\prime}\left(\alpha_{\kappa}\right)=u_{\kappa_{1}}, \quad \cdots, & F^{(n \kappa)}\left(\alpha_{\kappa}\right)=u_{\kappa n_{\kappa}}, \ldots, p, \\
& \kappa=1,2, \cdots, p=n, &
\end{array}
$$

where $u_{\kappa j}$ are given constants and $\alpha_{\kappa}$ are given constants within the unit circle.

Its uniqueness can be proved by just the same way as before. Its existence can be proved by considering the limit when $n_{\kappa}+1$ different $\alpha$ 's in the preceding theorem become equal to $\alpha_{\kappa}$, making the corresponding $n_{\kappa}+1$ values of the $u$ 's equal to the corresponding values of the function

$$
u_{\kappa_{0}}+\frac{1}{1 !} u_{\kappa_{1}}\left(x-\alpha_{\kappa}\right)+\frac{1}{2 !} u_{\kappa_{2}}\left(x-\alpha_{\kappa}\right)^{2}+\cdots+\frac{1}{n_{\kappa} !} u_{\kappa_{n_{k}}}\left(x-\alpha_{n}\right)^{n_{\kappa}} .
$$

In this limiting process, the corresponding sequence of expressions

$$
\prod_{i=1}^{n-1} E\left(x, t_{i}\right)
$$

must have a partial sequence which will converge to a definite expression of the same form, from which we get the required function by multiplying by some constant. Thus our theorem assumed in chapter III is completely proved.

\section{Remarks}

As has been said before, I have no practical method to obtain the expression (15) for $R(x)$ and $P(x)$. The only thing which $I$ have shown is some deeper relation between the required maximum and extremal.

I hope that the theorem of the chapter IV may be'proved purely algebraically, and the actual method of factorization determined if possible. If this could be done we can not only complete our present problem but also can prove my former theorem from which I deduced the uniqueness of our solution.

From the condition (29) we can easily calculate the values $v_{k j}$ for which

$$
\begin{aligned}
& G\left(\alpha_{\kappa}\right)= \pm v_{\kappa_{0}}, \quad G^{\prime}\left(\alpha_{\kappa}\right)= \pm v_{\kappa_{1}}, \quad \cdots, \quad G^{\left(n_{\kappa}\right)}\left(\alpha_{\kappa}\right)= \pm v_{\kappa_{k}} . \\
& \kappa=1,2, \cdots, p \text {, }
\end{aligned}
$$

where

$$
G(x)=\sqrt{F(x)},
$$

save for ambiguity of sign. 
Take a fixed upper or lower sign of $v_{x j}$ for each $k$ and construct a polynomial $G(x)$ at most of degree $n-1$, which satisfies the condition (30). It is uniquely determined by the method of interpolation.

If

$$
G(x)=c \prod_{i=1}^{e}\left(\bar{a}_{i} x-1\right), \quad 0 \leqq q \leqq n-1,
$$

thus obtained has all its zeros greater than 1 in absolute value, namely if all $|a|$ 's are less than 1 , then

$$
F(x)=c^{2} \prod_{i=1}^{q}\left(\bar{a}_{i} x-1\right)^{2}
$$

is the required expression for $F(x)$.

The problem treated by Professor Landau was fortunately one which belongs to this simple case.

Since we can not have two or more different expressions for the required $F(x)$, we obtain the following theorem of algebra.

Taking either the upper or the lower sign of (30) for each $k$, we get $2^{p}$ different functions $G(x)$, half of which differ only by signs from the remaining ones. Consider now these $2^{p-1}$ essentially different functions. There can not exist more than one function whose zeros are all numerically greater than 1 (strictly speaking, greater than the greatest of the $\left|\alpha_{i}\right|$ ).

We were considering the variable in the unit circle $|x|=1$ and the function whose modulus does not exceed 1 . But it can be very easily extended to the case where the variable is bounded in the domain $|x| \leqq r$ and the function in question is limited to be $|f(x)| \leqq R$.

\section{Examples}

We shall now consider a few examples.

1. To find the function $f(x)$, analytic and numerically not exceeding 1 for $|x| \leqq 1$, which makes

$$
\left|f(\alpha)+A f^{\prime}(\alpha)\right|
$$

greatest, $\alpha$ being a given constant numerically less than 1 .

Here we have

$$
\begin{gathered}
p=1, \quad n_{1}=1, \quad \alpha_{1}=\alpha, \\
P(x)=(x-\alpha)^{2}, \quad Q(x)=\frac{A+(x-\alpha)}{2 \pi i}, \\
R(x)=\frac{1}{2 \pi i} A(\alpha \bar{\alpha}-1)^{2}+\frac{1}{2 \pi i}(\alpha \bar{\alpha}-1)\{(\alpha \bar{\alpha}-1)+2 A \bar{\alpha}\}(x-\alpha) .
\end{gathered}
$$

There are only two cases, namely $m=1$ or $m=0$. First assuming that $m=1$ we put

$$
R(x)+(x-\alpha)^{2} \psi(x)=c(\bar{a} x-1)^{2}=c\{\bar{a}(x-\alpha)+\bar{a} c-1\}^{2} ;
$$


then we get

$$
\begin{aligned}
\frac{1}{2 \pi i} A(\alpha \bar{\alpha}-1)^{2} & =c(\bar{a} \alpha-1)^{2} \\
\frac{1}{2 \pi i}(\alpha \bar{\alpha}-1)\{(\alpha \bar{\alpha}-1)+2 A \bar{\alpha}\} & =2 c \bar{a}(\bar{a} \alpha-1) .
\end{aligned}
$$

Hence

$$
\frac{\bar{a} \alpha-1}{2 \bar{a}}=\frac{A(\alpha \bar{\alpha}-1)}{(\alpha \bar{\alpha}-1)+2 A \bar{\alpha}}
$$

or

$$
\bar{a}=\frac{(\alpha \bar{\alpha}-1)+2 A \bar{\alpha}}{\alpha(\alpha \bar{\alpha}-1)+2 A}
$$

If this value of $\bar{a}$ is numerically less than 1 , namely

$$
A>\frac{1-|\alpha|^{2}}{2}
$$

then the required extremal is

$$
\begin{aligned}
f(x) & =e^{i \theta} \frac{x-a}{\bar{a} x-1} \\
& =e^{i \theta} \frac{x-\frac{(\alpha \bar{\alpha}-1)+2 \bar{A} \alpha}{\bar{\alpha}(\alpha \bar{\alpha}-1)+2 \bar{A}}}{\frac{(\alpha \bar{\alpha}-1)+2 A \bar{\alpha}}{\alpha(\alpha \bar{\alpha}-1)+2 A}-1} .
\end{aligned}
$$

If

$$
A \leqq \frac{1-|\alpha|^{2}}{2},
$$

then $m$ must be zero and hence the required function is

$$
f(x)=e^{i \theta} \text {. }
$$

2. To find the function, analytic and numerically not greater than 1 for $|x| \leqq 1$, which makes $|f(\alpha)-f(-\alpha)|$ greatest, $\alpha$ being a given constant such that $0<|\alpha|<1$.

Here we have

$$
\begin{array}{lr}
n=2, \quad \alpha_{1}=\alpha, & \alpha_{2}=-\alpha, \\
P(x)=x^{2}-\alpha^{2}, & Q(x)=\frac{\alpha}{\pi i} .
\end{array}
$$

$R(x)$ is the remainder of $\alpha\left(1-\bar{\alpha}^{2} x^{2}\right) /(\pi i)$ divided by $x^{2}-\alpha^{2}$. So the function $F(x)=R(x)+P(x) \psi(x)$ has the property that

$$
F(\alpha)=F(-\alpha)=\alpha\left(1-\bar{\alpha}^{2} \alpha^{2}\right) /(\pi i) \text {. }
$$


Hence if we put

$$
F(x)=c(\bar{a} x-1)^{2},
$$

assuming that $m=1$, we must have

$$
\frac{F(\alpha)}{F(-\alpha)}=\left(\frac{\bar{a} \alpha-1}{\bar{a} \alpha+1}\right)^{2}=1,
$$

or

$$
\bar{a}=0 \text {. }
$$

Thus $\bar{a}$ is numerically less than 1 , and hence the required extremal is

$$
f(x)=e^{i \theta} \frac{x-a}{\bar{a} x-1}=e^{i \rho} x .
$$

\section{Higher Normal Schoor,}

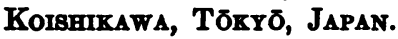

